\title{
PROPAGACIÓN Y DIAGNÓSTICO DE REGENERACIÓN NATURAL DE ALGUNAS ESPECIES MADERABLES EMPLEADAS POR LA COMUNIDAD INDÍGENA DE MOCAGUA (Parque Nacional Natural Amacayacu. Amazonas-Colombia) ${ }^{1}$
}

Palabras clave: Comunidad indígena, investigación participativa, conocimiento local, ensayos de producción, regeneración natural, Amazonas.

Key words: Community indigenous, participant methodology, local knowledge, production of plants, natural regeneration, Amazon.

\section{RESUMEN}

Este proyecto se realizó en la comunidad indígena Ticuna de Mocagua, en el sur del Trapecio Amazónico colombiano. Evaluó las posibilidades de propagar Palisangre (Brosimun rubescens), Acapú (Minquartia guianensis), Aceituno (Vitex sp.), Ojé (Ficus insipida) y Quinilla (Manilkara bidentata), especies maderables que están siendo usadas por la comunidad y presentan algún grado de escasez. En el marco de una metodología participativa, se incluyeron principios y saberes ancestrales indígenas, al mismo tiempo que se aplicaron técnicas y conocimientos manejados por la comunidad científica. Con los resultados obtenidos se identificó parcialmente el mejor método de propagación para aumentar las poblaciones de las especies en estudio, especies de alto interés local, y además, se avanzó en el objetivo de utilizar sosteniblemente los recursos naturales dentro del Resguardo Indígena. El resultado más importante que se obtuvo con el desarrollo de esta investigación fue el planteamiento, por parte de los investigadores indigenas locales, de iniciativas relativas a la organización de nuevos proyectos que continúen y mejoren los resultados obtenidos, buscando realizar ensayos de plantación y enriquecimiento forestal,

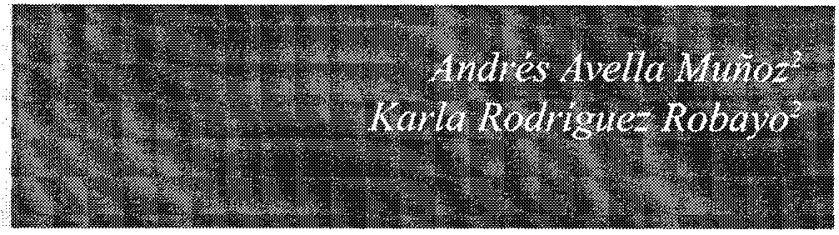

en jornadas de siembras comunitarias orientadas a la recuperación de zonas degradadas y al mejoramiento de las poblaciones actuales de árboles con importancia local.

\section{ABSTRACT}

This project was carried out in the Mocagua's community indigenous, in the south of Colombian Amazon. It evaluated the possibility of spreading Palisangre (Brosimun rubescens), Acapú (Minquartia guianensis), Aceituno (Vitex sp.), Ojé (Ficus insipida) and Quinilla (Manilkara bidentata); woody species that are being used by the community and they have some degree of shortage. In the framework of a participant methodology was included as indigenous ancestral knowledge as techniques and knowledge managed by the scientific community. The outcomes permit to identify the better way for spreading of increasing the population of study in species, and besides, it advanced in the purpose of using sustainable the natural resources of Indigenous Resguardo.

The most important outcome was the emergence in the local indigenous researcher of own ideas and proposal that keeping on and improving the obtained results, looking for

1 Proyecto de Investigación financiado por Fundación Tropenbos Internacional - Colombia

2 Ingenieros Forestales. Becarios Fundación Tropenbos-Colombia. eaavella@yahoo.com; karlajuliana@hotmail.com 
making in communal working day, plantation and enrichment forester for recovering areas and the tree's populations affected.

\section{INTRODUCCIÓN}

El Parque Nacional Natural Amacayacu (PNNA) hace parte del Sistema de Parques Nacionales Naturales de Colombia, localizado en el Trapecio Amazónico, protege una extensión total de 293.500 ha del ecosistema de selva húmeda tropical. Uno de sus objetivos de manejo es conservar en el mejor estado natural posible, muestras representativas de los ecosistemas propios del trapecio. El PNNA se encuentra traslapado con tres resguardos indígenas pertenecientes a la Etnia Tikuna (Suárez et al., 1998), quienes según Arias (1997); basan su economía en actividades de caza, pesca, agricultura de subsistencia, y una actividad artesanal que recientemente se ha impulsado como una buena estrategia de comercio. Por lo anterior, se hace vital la existencia de una armonía entre las prácticas culturales empleadas por las comunidades indígenas para satisfacer sus necesidades y los objetivos de conservación de los recursos naturales que tiene el PNNA.

Las materias primas necesarias para la construcción de casas y elaboración de artesanías se obtienen directamente del bosque natural bajo prácticas de manejo tradicionales que se consideran sostenibles. Sin embargo, existen opiniones de investigadores, artesanos y habitantes de las mismas comunidades, en torno a la progresiva escasez de las especies utilizadas como materias primas (Franco, 2002; Panduro com. pers.).

La investigación silvicultural en métodos de propagación y manejo de especies nativas es un paso necesario para el uso sostenible de nuestra biodiversidad. No obstante, un aspecto que por lo general no se incluye en este campo de investigación, es el amplio saber que tienen las comunidades indígenas y rurales, toda vez que son ellos los que han convivido y observado constantemente los patrones y estrategias de reproducción natural de las especies.

Es interesante y bastante útil lograr reunir el conocimiento tradicional con el de la ciencia occidental y así construir propuestas que aporten al uso sostenible de los recursos naturales y que, como lo sugieren Folke et al., (2002), puedan contrarrestar algunas de las actuales crisis relacionadas con el manejo convencional de los recursos naturales.

Sin embargo, este tipo de iniciativas deben surgir de igual manera al interior de las comunidades, responder a procesos endógenos que, como señalan Suárez et al., (op. cit.), promuevan el desarrollo de alternativas "localmente pensadas y realizadas", donde la misma comunidad indígena sea partícipe activo desde la concepción, formulación y ejecución de los proyectos, en un intento de respeto a su opinión, criterio y sentido de su cultura.

A partir de la conformación de un grupo de investigación conjunto, entre actores locales y externos, el presente proyecto realizó ensayos de propagación y diagnósticos de regeneración natural de algunas especies forestales de interés local en la comunidad indígena de Mocagua. La investigación se basó en una metodología participativa, que permitió incluir los principios, saberes y conocimientos ancestrales indígenas, al mismo tiempo que se aplicaron los conocimientos y técnicas manejadas por la comunidad científica.

\section{METODOLOGÍA}

La Investigación Acción Participativa fue la metodología de investigación que se siguió principalmente, se utilizaron herramientas de trabajo comunitario que buscaban generar una real convocatoria en torno al objetivo del proyecto $\mathrm{y}$, por ende, una apropiación del mismo. La "Investigación participativa" permitió in- 
cluir los principios, saberes y conocimientos ancestrales indígenas, al mismo tiempo que se aplicaron los conocimientos y técnicas manejadas por la comunidad científica, en una forma de diálogo de saberes.

El acompañamiento a actividades como el trabajo en la chagra, la pesca, la caza y la recolección de materias primas para elaborar artesanías (La Rotta, 1987; Garzón y Macuritofe, 1992), permitieron complementar la información recopilada en las reuniones y jornadas de campo; además, de esta manera se generó una relación de confianza entre los miembros del grupo.

\section{RESULTADOS}

\section{CONOCIÉNDONOS ACTIVIDADES INICIALES}

En esta parte se presentan algunas de las actividades con las cuales se inició el acercamiento a la comunidad; realmente éstas se convir- tieron en resultados de investigación, en cuanto permitieron el inicio del trabajo en grupo, el reconocimiento de los saberes que cada uno de los integrantes del grupo tenía y principalmente facilitaron la creación de un clima de confianza y amistad.

\section{- Conformación del Grupo de trabajo}

Inicialmente se presentó la propuesta de investigación al Grupo de Trabajo en Investigación (GTI) y allí se decidió que se llevaría a cabo en la comunidad de Mocagua. La propuesta fue presentada a toda la comunidad indígena para que ella diera su opinión acerca del trabajo y se inscribieran las personas interesadas en conformar el grupo de investigación del proyecto. Los días siguientes se hicieron reuniones concretando el grupo, conociéndonos, aclarando la forma de trabajo que más nos convenía, y planeando las primeras actividades a realizar. El grupo de investigación quedó conformado por las personas que se reseñan en la Tabla 1.

Tabla 1. Integrantes del grupo de investigación.

\begin{tabular}{|ccll|}
\hline \multicolumn{1}{c}{ NOMBRE } & EDAD & ETNIA & OCUPACIóN \\
\hline Leonel Pandero & 54 & Tikuna & Cazador, investigador \\
Consuelo Santos & 44 & Tikuna & Hogar, artesana y chagra \\
Pio Cayetano & 37 & Tikuna & Cazador, agricultor, constructor \\
Matilde Santos & 39 & Tikuna & Chagra, artesana y hogar \\
Sergio León & 44 & Tikuna & Artesano, pescador \\
Francisco Rojas & 48 & Mestizo & Artesano, Pescador \\
Francisco Rojas (hijo) & 24 & Mestizo & Pescador \\
Donelia Olanda & 63 & Mestiza & Casa, chagra \\
Victoria Meléndez & 25 & Mestiza & Casa, chagra \\
Karla J. Rodríguez & 22 & Blanca & Ing. Forestal \\
Andrés Avella M & 24 & Blanca & Ing. Forestal \\
\hline
\end{tabular}

- Selección de especies

Seguidamente, con el grupo de trabajo se definieron las especies que por su dificultad de consecución e importancia socioeconómica se consideraban prioritarias para propagar. En la Tabla 2 se presentan las especies seleccionadas. 
Tabla 2. Especies seleccionadas para realizar ensayos de propagación y diagnóstico de regeneración natural.

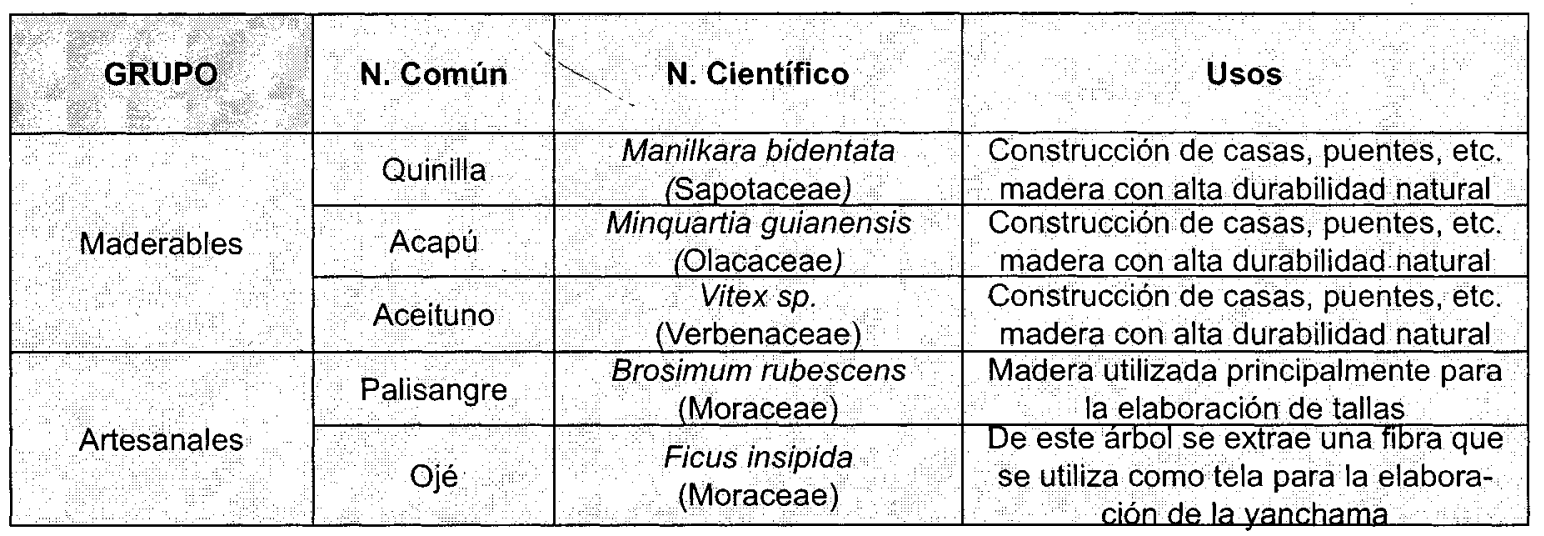

- Haciendo casa de plantas (Construcción del vivero)

La elaboración de un vivero requiere tener en cuenta una serie de factores que garanticen un adecuado desarrollo de las plántulas y del material vegetal que se tendrá en ese lugar. Como lo indica Trujillo (1999), de una acertada elección del sitio, depende en buena medida el éxito o el fracaso que pueda tener el vivero.

Éstas y otras consideraciones técnicas eran conocidas por los investigadores locales, por lo cual la elección del sitio se convirtió en un enriquecedor diálogo de saberes donde todos discutíamos ideas sobre el lugar ideal para realizar nuestro vivero. Finalmente, y luego de evaluar en campo algunos posibles terrenos, se decidió construir el vivero en un rastrojo de 8 años, propiedad de Sergio León. El terreno se encuentra dentro de una zona de chagras indígenas de tierra firme no inundable, con un área aproximada de $250 \mathrm{~m}^{2}$, de forma semirrectangular, plano y cerca a un caño de aguas limpias de carácter permanente.

Se construyeron cuatro eras, cada una de 16 $\mathrm{m}^{2}$, aproximadamente; dos se destinaron a los ensayos de propagación asexual (estacas), una a la propagación sexual (semillas) y una para las plántulas provenientes del bosque. El vivero contaba así mismo con un espacio suficiente para el depósito de los materiales requeridos y para la manipulación de los mismos.

\section{- Evaluación de variables}

Como el objetivo era determinar bajo qué condiciones se desarrollan mejor los individuos de las especies seleccionadas, los investigadores locales señalaron en primera instancia que era necesario dejar alrededor y dentro del vivero algunos árboles grandes y techar muy bien las eras con hojas de Canangucho (Mauritia flexuosa), Bacaba (Oneocarpus sp.) y Shapaja (Scheelea sp.) para regular la cantidad de luz que llegaría al vivero, debido a que todas las especies seleccionadas (excepto el Ojé) pertenecían al bosque maduro.

Los investigadores externos queríamos evaluar diferentes tipos de sustratos debido a las características que presentan los suelos de la región: pesados, arcillosos, de regular a mal drenaje, además de las. señaladas por IGAC (1995): altas concentraciones de aluminio, baja fertilidad y contenidos bajos de carbón orgáni$\mathrm{co}$, nitrógeno y fósforo.

Por tal razón era necesario que el grupo de investigación identificara algunos posibles materiales que mejoraran las características estructurales y de textura del suelo; durante esta actividad se mostró el gran aporte conceptual 
y técnico proveniente del conocimiento local. Por ejemplo, los investigadores externos pensábamos utilizar la arena de las playas del río para mejorar la aireación y el drenaje del suelo, pero los investigadores locales, basados en su gran conocimiento tradicional, entendían lo que queríamos hacer, pero nos comentaban que esa arena "era puro barro que quemaba muy fácil las plantas"; entonces nos propusieron utilizar un tipo de arena especial que se encuentra en algunas partes de la Isla Mocagua y que podría utilizarse satisfactoriamente en la elaboración del sustrato.

También al mencionarles la necesidad de mejorar la fertilidad del suelo, los investigadores locales propusieron utilizar el "abono de palo muerto", que tiene características favorables: suelto, con una alta cantidad de materia orgánica, con capacidad de retener humedad pero de buen drenaje, características muy similares a la tierra negra que se encuentra en climas fríos. Fue este material la base principal de los sustratos para germinación de semillas y trasplante de regeneración natural.
Un aspecto en el cual coincidimos investigadores externos y locales fue la necesidad de evaluar el grosor ideal (diámetro) que deberían tener las estacas, para desarrollar un sistema radicular y vegetativo adecuado. Las estacas juveniles poseen una gran capacidad de regeneración de tejidos pero cuentan con menor cantidad de nutrientes almacenados. Por otro lado, las estacas con diámetros mayores tienen mayor cantidad de nutrientes, pero debido a su mayor edad, se supone una menor capacidad de enraizamiento. Las variables a evaluar en los ensayos de semillas se definirían según el tipo de fruto y semilla que tendría cada una de las especies seleccionadas.

\section{SEMBRANDO RAMAS - Ensayos de propagación asexual -}

A continuación se presenta una tabla resumen de las principales actividades llevadas a cabo con el fin de realizar los ensayos de propagación. En cada una de las actividades se incluyeron técnicas manejadas por los investigadores locales y por los externos (Tabla 3).

Tabla 3. Principales actividades para realizar los ensayos de propagación

\begin{tabular}{|c|c|}
\hline ACTIVIDAD & DESCRIPCIÓN \\
\hline $\begin{array}{l}\text { Buscando las mejores madres... } \\
\text { selección de fuentes semilleras }\end{array}$ & $\begin{array}{l}\text { Los recorridos se organizaron con los investigadores locales } \\
\text { que tuvieran más conocimiento de la zona. Se inició con re- } \\
\text { corridos para buscar los posibles árboles madres; se tuvieron } \\
\text { en cuenta características como fustes o troncos rectos, sin } \\
\text { bifurcaciones tempranas, copas amplias y sanidad. }\end{array}$ \\
\hline $\begin{array}{l}\text { Recolección de ramas, } \\
\text { Ascenso al árbol y corte de estacas }\end{array}$ & $\begin{array}{l}\text { El ascenso a los árboles madres se realizaba con técnicas } \\
\text { tradicionales: utilización de bejucos, árboles aledaños y fibras } \\
\text { de cortezas }\end{array}$ \\
\hline $\begin{array}{l}\text { Preparando los palitos. } \\
\text { Acondicionamiento de estacas }\end{array}$ & $\begin{array}{l}\text { Dimensionamiento final, clasificación y desinfección de } \\
\text { estacas }\end{array}$ \\
\hline $\begin{array}{l}\text { Sembrando y } \\
\text { Siembra }\end{array}$ & $\begin{array}{l}\text { Esta actividad se realizó únicamente conlas mujeres, ya } \\
\text { que es considerado entre los indígenas, que la mujer por ser } \\
\text { fecunda tiene la capacidad de generar vida al sembrar, de he- } \\
\text { cho es ella la encargada de la siembra en la chagra, realizan- } \\
\text { do el hombre únicamente el trabajo de la limpla. } \\
\text { La frecuencia de riego variaba dependiendo del clima, regan- } \\
\text { dose dos veces en dias calurosos y una vez en dias lluviosos. }\end{array}$ \\
\hline
\end{tabular}


El ensayo se llevó a cabo a través de un diseño experimental en Bloques Completos al Azar (BCA) con tres repeticiones. El factor de diferenciación fue el sustrato, en el bloque 1 se utilizó una mezcla de 1 Suelo +1 Arena +1 Ase- rrín, mientras que en el bloque 2 se utilizó 1,5 Suelo + 1Arena. La unidad muestreal se conformó con 10 estacas por tratamiento, 240 estacas por especies y en total 1200 estacas. Los factores, niveles y tratamientos se presentan en la Tabla 4.

Tabla 4. Factores, niveles y tratamientos del ensayo de propagación asexual.

\begin{tabular}{|c|c|c|c|}
\hline $\begin{array}{c}\text { BLOQUE } \\
\text { Factor de diferenciación }\end{array}$ & \multicolumn{2}{|l|}{ NIVELES } & \multirow{2}{*}{ Tto } \\
\hline \multirow{5}{*}{ 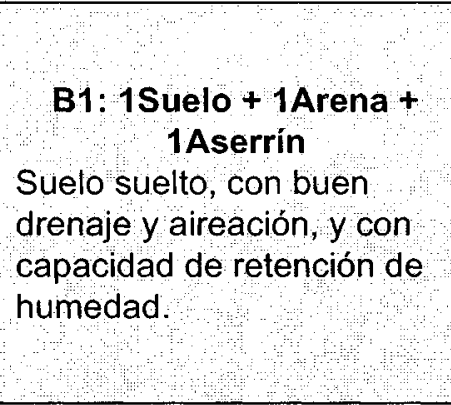 } & Diámetro (cm) & Hormona (ppm) & \\
\hline & \multirow{2}{*}{$\begin{array}{l}\qquad 2<\varnothing<4 \\
\text { Estacas con mayor cantidad de } \\
\text { nutrientes; debido a su mayor edad, } \\
\text { se supone una menor capacidad de } \\
\text { enraizamiento }\end{array}$} & 5000 & 1. \\
\hline & & Testigo & 2 \\
\hline & \multirow{2}{*}{$\begin{array}{l}\qquad \varnothing<2 \\
\text { Estacas juveniles pero con menor } \\
\text { cantidad de nutrientes almacenados }\end{array}$} & 5000 & 3 \\
\hline & & Testigo & 4 \\
\hline \multirow{4}{*}{$\begin{array}{l}\text { B2: } 1,5 \text { Suelo + } 1 \text { Arena } \\
\text { Suelo similar al típico de la } \\
\text { zona, se agrega arena para } \\
\text { mejorar el drenaje. }\end{array}$} & \multirow{2}{*}{$\begin{array}{l}\qquad 2<\varnothing<4 \\
\text { Similares características a las ante- } \\
\text { riormente nombradas. }\end{array}$} & 5000 & 1 \\
\hline & & Testigo & 2 \\
\hline & \multirow{2}{*}{$\begin{array}{l}\qquad \varnothing<2 \\
\text { Similares características a las ante- } \\
\text { riormente nombradas. }\end{array}$} & 5000 & 3 \\
\hline & & Testigo & 4 \\
\hline
\end{tabular}

Los principales indicadores que se tomaron en cuenta para el éxito de los ensayos de propagación vegetativa fueron la formación de rebrotes foliares, posteriormente hojas y la formación de callos, posteriormente raíces. El ensayo duró establecido un mes, tiempo realmente corto para la obtención de resultados concretos, diferentes autores cômo Hartman \& Kester (1987), Navarro \& Penman (1997), citan que el tiempo necesario para el completo establecimiento de las estacas varía entre uno y tres años.

Sin embargo, este trabajo se constituye en un real indicador del éxito o fracaso de la propagación vegetativa de las especies analizadas, ya que aunque no cuenta con un seguimiento prolongado, ofrece resultados preliminares en los cuales hay presencia de los indicadores determinantes para el establecimiento de las estacas. A continuación se presentan los resultados de reproducción por estacas de cada una de las especies trabajadas.
-Aceituno (Vitex sp.)

Ésta es la especie que presentó mejores resultados y en la que más confianza tenía el grupo de trabajo, pues los investigadores locales comentaban que entre el monte, al encontrar troncos apeados de aceituno, siempre presentaban algún rebrote. En cada especie los resultados se analizaron según las variables estudiadas (diámetros, inmersión en hormonas y sustrato) y a los tratamientos (T1, T2, T3 y T4). Para este ensayo las mayores diferencias de crecimiento se presentaron entre sustratos, en el sustrato 1 (Isuelo + larena + laserrín) 104 estacas formaron callo (87\%), mientras en el sustrato $2(1,5$ suelo + larena), lo formaron solamente $43(36 \%)$ (Tabla 5).

De acuerdo con los tratamientos, los mejores resultados $(97-100 \%$ de enraizamiento) se obtuvieron con T1 y T3 en el Bloque 1, es decir que en el desarrollo radicular es indiferente el 
diámetro; sin embargo, la inmersión en hormonas $(5000 \mathrm{ppm})$ y la siembra en el sustrato 1 son indispensables para obtener estos altos porcentajes (Tabla 5 y Figura 1). Por otra par- te, según el análisis estadístico, no se encontraron diferencias significativas entre los tratamientos, reflejándose la gran capacidad de esta especie para reproducirse asexualmente.

Tabla 5. Resumen de los resultados obtenidos para aceituno (Vitex $s p$.) en los ensayos de propagación vegetativa.

\begin{tabular}{|l|r|r|r|r|}
\hline \multicolumn{1}{|c|}{ VARIABLES } & \multicolumn{2}{|c|}{ Callo } & \multicolumn{2}{c|}{ Rebrotes } \\
\cline { 2 - 5 } & $\mathrm{N}^{0}$ & $\%$ & \multicolumn{1}{|c|}{$\mathrm{N}^{0}$} & $\%$ \\
\hline Diam: $2<\varnothing<4 \mathrm{~cm}$ & 77 & 64 & 77 & 64 \\
\hline Diam: $\varnothing<2 \mathrm{~cm}$ & 70 & 58 & 73 & 61 \\
\hline Con Auxinas & 71 & 59 & 80 & 67 \\
\hline Sin Auxinas & 76 & 63 & 70 & 58 \\
\hline Sustrato 1 & 104 & 87 & 78 & 65 \\
\hline Sustrato 2 & 43 & 36 & 72 & 60 \\
\hline
\end{tabular}

\begin{tabular}{|c|r|r|}
\hline \multicolumn{3}{|c|}{ Formacion Callo } \\
\hline Tto & B1 & \multicolumn{1}{|c|}{ B2 } \\
\cline { 2 - 3 } & $(\%)$ & $(\%)$ \\
\hline T1 & 100 & 30 \\
\hline T2 & 76,7 & 50 \\
\hline T3 & 96,7 & 10 \\
\hline T4 & 73,3 & 53,3 \\
\hline
\end{tabular}
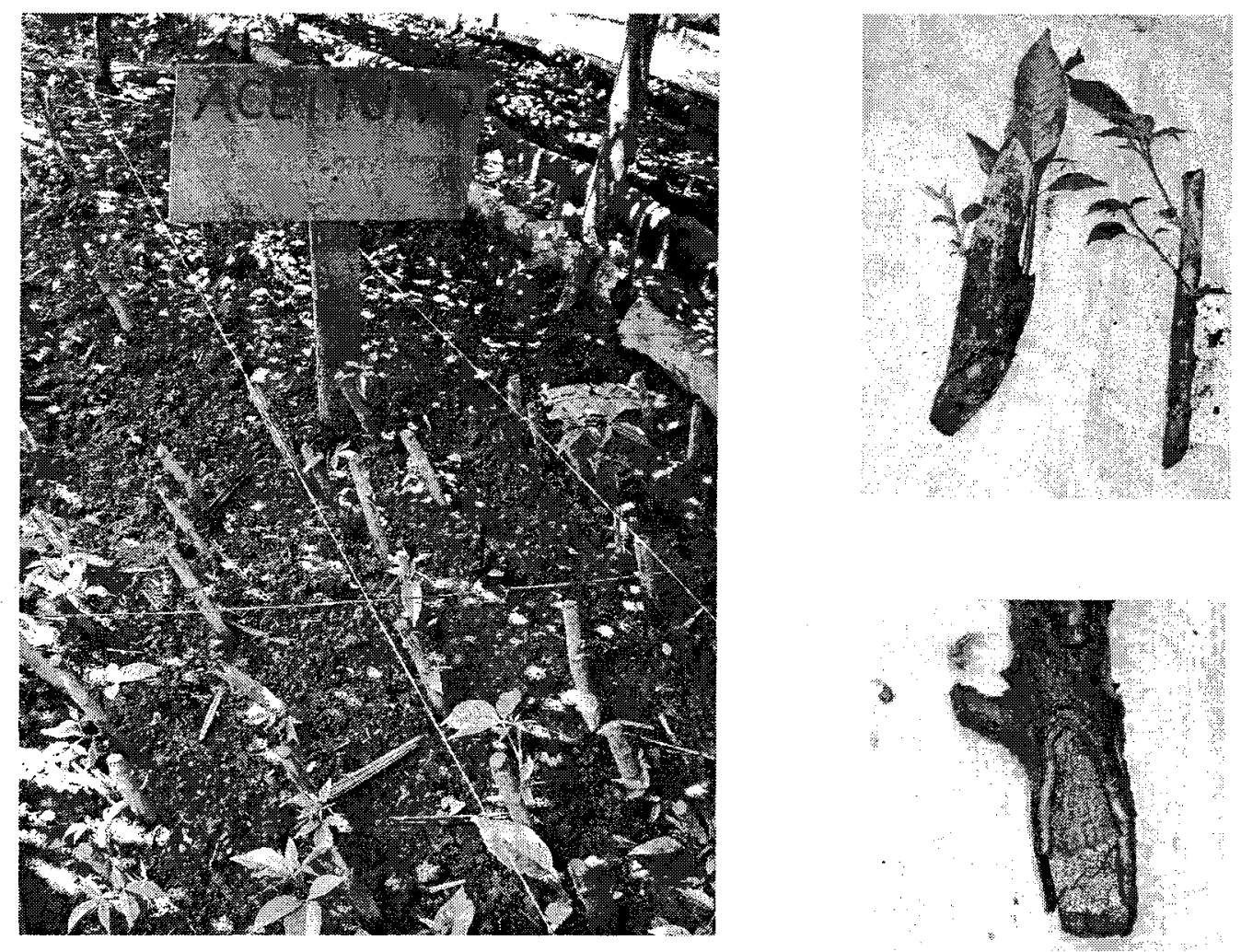

Figura 1. Resultados obtenidos para aceituno (Vitex sp.) en los ensayos de propagación vegetativa. Foto N. Lozano. 2004 


\section{- Palisangre (Brosimum rubescens)}

En el $B$. rubescens, las diferencias más grandes se presentaron entre diámetros, pues 33 estacas con diámetro entre $2-4 \mathrm{~cm}$ formaron callo $(28 \%)$, mientras que del diámetro inferior $(<2 \mathrm{~cm})$ tan sólo 2 lo formaron (2\%) (Tabla 6).
Lo anterior se confirma con el análisis estadístico (Anexo 1), según la prueba de Tukey los mejores tratamientos (30\% de enraizamiento) son $\mathrm{Tl}$ (estacas de diámetro entre $2-4 \mathrm{~cm}$, con inmersión en hormonas), seguido de T2 (estacas de diámetro menor a $2 \mathrm{~cm}$, sin inmersión en hormonas).

Tabla 6. Resumen de los resultados obtenidos para palisangre

(B. rubescens) en los ensayos de propagación vegetativa.

\begin{tabular}{|l|r|r|r|r|}
\hline \multirow{2}{*}{\multicolumn{1}{c|}{ VARIABLES }} & \multicolumn{2}{|c|}{ Callo } & \multicolumn{2}{c|}{ Raíces } \\
\cline { 2 - 5 } & $N^{0}$ & $\%$ & $N^{0}$ & $\%$ \\
\hline Diam: $2<\varnothing<4 \mathrm{~cm}$ & 33 & 28 & 10 & 8 \\
\hline Diam: $\varnothing<2 \mathrm{~cm}$ & 2 & 2 & 0 & 0 \\
\hline Con Auxinas & 20 & 17 & 7 & 6 \\
\hline Sin Auxinas & 15 & 13 & 3 & 3 \\
\hline Sustrato 1 & 19 & 16 & 0 & 0 \\
\hline Sustrato 2 & 16 & 13 & 10 & 8 \\
\hline
\end{tabular}

\begin{tabular}{|c|r|r|}
\multicolumn{3}{|c|}{ Formacion Callo } \\
\hline \multirow{2}{*}{ Tto } & B1 & B2 \\
\cline { 2 - 3 } & $(\%)$ & $(\%)$ \\
\hline T1 & 30 & 33,3 \\
\hline T2 & 30 & 16,7 \\
\hline T3 & 0 & 3,3 \\
\hline T4 & 3,3 & 0 \\
\hline
\end{tabular}

Así mismo, se observaron diferencias entre los dos sustratos, se encontró la particularidad que en el sustrato 2 hubo formación de raíces, mientras que en el 1 no; posiblemente esto se deba a que el segundo sustrato es más similar al característico de su hábitat natural, ya que al no contener aserrín, se convierte en un sustrato más arcilloso e impermeable, que de acuerdo con Palacios (Com. pers. 2003) es una exigencia de esta especie para su desarrollo (Figura 2).

Es necesario comentar que de los cinco géneros trabajados, Brosimum fue el único del cual se encontraron ensayos realizados en propagación por estacas. Sin embargo, los ensayos fueron realizados para la especie sande (B. utile), en 1983 por Cortés y en 1986 por Díaz, en los cuales a los quince y cuarenta días, respectivamente, de plantadas la estacas obtuvieron un $100 \%$ de mortalidad.
- Quinilla (Manilkara bidentata) y Ojé (Ficus insipida)

En la quinilla es posible observar diferencias entre diámetros, ya que el $15 \%$ de las estacas con diámetro superior formaron callo, mientras que de las estacas con diámetro menor solo el $4 \%$ lo formaron. Entre sustratos la diferencia es aún más grande puesto que el $19 \%$ de las estacas del sustrato 1 formaron callo, mientras que en el sustrato 2 ninguna lo hizo (Tabla 7). De acuerdo con los resultados obtenidos por tratamientos, el mejor es T2 (40\%) en el bloque 1, es decir estacas con diámetro entre 2 y $4 \mathrm{~cm}$, sin inmersión en auxinas, en el sustrato 1. Sin embargo, teniendo en cuenta que no se obtuvo resultados para el bloque 2, en el análisis estadístico no se identificaron diferencias entre tratamientos. Por otra parte, es necesario anotar que en la quinilla se observó en un alto número de estacas, la presencia de lenticelas hinchadas, indicador para la formación de raíces. 

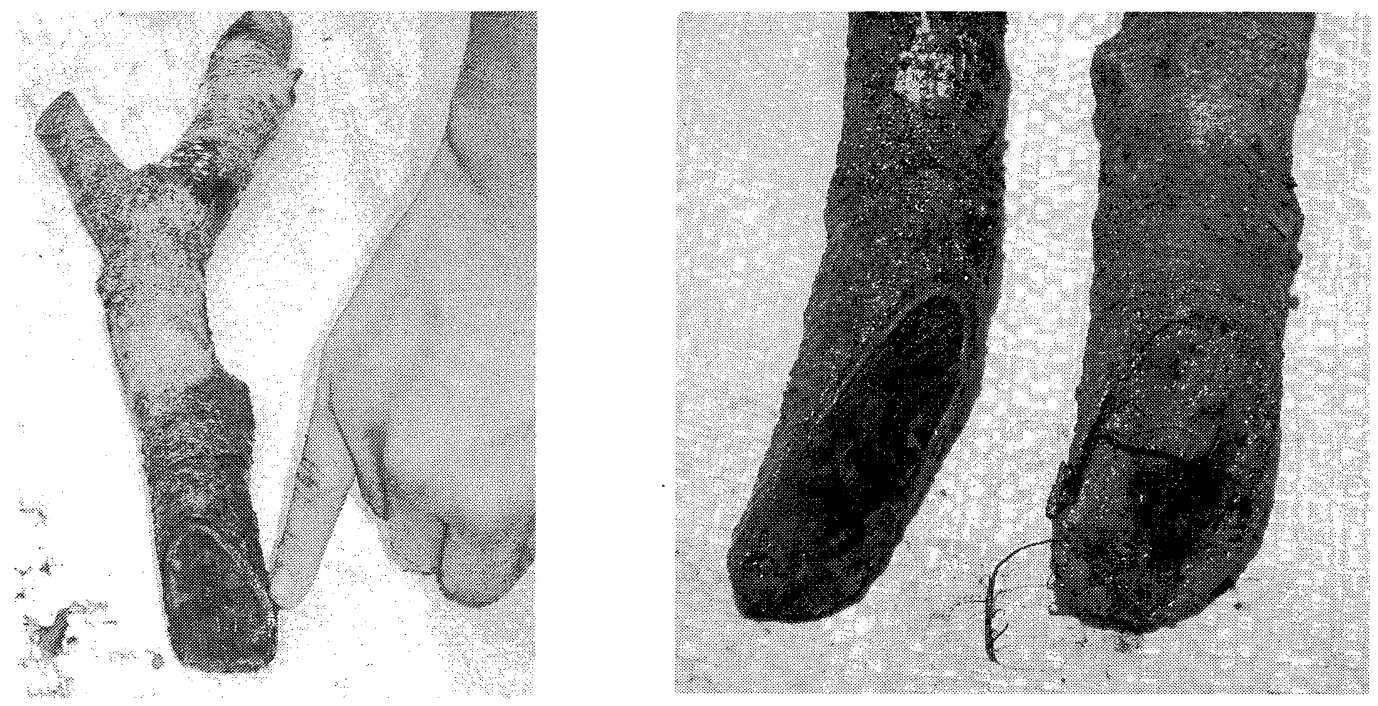

Figura 2. Resultados obtenidos para palisangre (B. rubescens) en los ensayos de propagación vegetativa. Foto N. Lozano. 2004.

Tabla 7. Resumen de los resultados obtenidos para quinilla

(M. bidentata) en los ensayos de propagación vegetativa.

\begin{tabular}{|l|r|r|r|r|r|r|r|}
\hline \multirow{2}{*}{ VARIABLES } & \multicolumn{2}{|c|}{ Callo } & \multicolumn{2}{c|}{ Rebrotes } \\
\cline { 2 - 7 } & $\mathrm{N}^{0}$ & $\%$ & $\mathrm{~N}^{0}$ & $\%$ \\
\hline Diam: $2<\varnothing<4 \mathrm{~cm}$ & 18 & 15 & 0 & 0 \\
\hline Diam: $\varnothing<2 \mathrm{~cm}$ & 5 & 4 & 0 & 0 \\
\hline Con Auxinas & 9 & 8 & 0 & 0 & \multicolumn{2}{c|}{ Formacion Callo } \\
\hline Sin Auxinas & 14 & 12 & 0 & 0 & $\mathrm{~B} 1$ & $\mathrm{~B} 2$ \\
\hline Sustrato 1 & 23 & 19 & 0 & 0 & $(\%)$ & $(\%)$ \\
\hline Sustrato 2 & 0 & 0 & 0 & 0 \\
\hline
\end{tabular}

En el ojé se obtuvo únicamente la formación de rebrotes foliares en un bajo número de estacas; el inconveniente que presentó esta especie, debido a su carnosidad, altos contenidos de líquidos y a la poca permeabilidad del suelo, fue que sufrió un ataque de hongos, haciéndose necesario desmontar el ensayo para prevenir la infección de las otras especies. Sin embargo, el ojé fue la especie que primero arrojó resultados, y seguramente mejorando las condiciones del suelo y ampliando el área del ensayo se pueden controlar este tipo de enfermedades (Figura 3).

\section{LAS PEPAS Ensayos de propagación sexual}

Caminando entre el bosque, en la búsqueda de árboles madres y fuentes semilleras, se observó que para ninguna de las especies trabajadas era tiempo de fructificación. Lo más probable es que el tiempo de trabajo en campo no haya coincidido con la etapa de fructificación de las especies en estudio, ya que el haber observado regeneración natural, es un indicador de la producción de semilla viable dentro del bosque. Por estas razones los ensayos de propagación sexual no se pudieron realizar; a pesar de esto, se logró construir un conocimiento en torno a los frutos y semillas de las especies trabajadas (Tabla 8). 

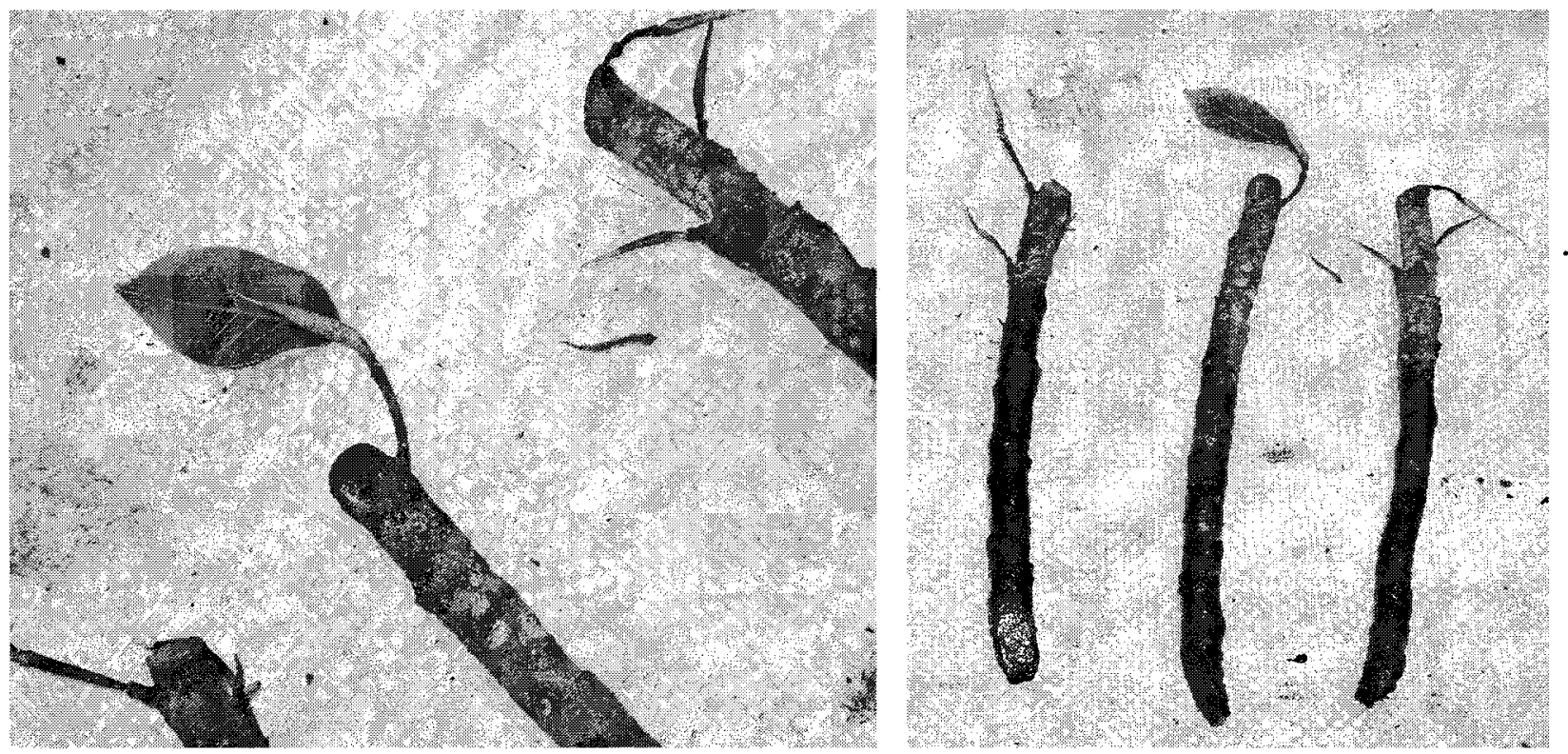

Figura 3. Resultados obtenidos para ojé (F. insipida) en los ensayos de propagación vegetativa. Foto N. Lozano. 2004.

Tabla 8. Tiempos de fructificación de las especies en estudio

\begin{tabular}{|cl|}
\hline ESPECIE & \multicolumn{1}{c|}{ Características } \\
\hline PALISANGRE & $\begin{array}{l}\text { Conocimiento de los frutos y semillas con el apoyo del grupo de investigación U. } \\
\text { Nacional - U. Distrital (Leticia, Amazonas). Gran cantidad de semilla vana debajo } \\
\text { de los árboles madres. Al ascender a los árboles no se observó ni fruto ni semilla }\end{array}$ \\
\hline QUINILLA & $\begin{array}{l}\text { Tiempo de fructificación entre noviembre y enero. Frutos muy apetecidos para el } \\
\text { consumo animal y humano }\end{array}$ \\
\hline ACAPÚ & Tiempo de fructificación entre septiembre a noviembre \\
\hline OJÉ & Tiempo de fructificación entre febrero y marzo \\
\hline ACEITUNO & Tiempo de fructificación entre noviembre y enero \\
\hline
\end{tabular}

\section{VISITANDO SUS CRÍAS. Diagnósticos de Regeneración Natural}

Los principios ecológicos de "Competencia Intraespecifica" y "Distribución diamétrica" nos motivaron a investigar la regeneración natural de las especies seleccionadas y a evaluar si era posible obtener del mismo bosque el material vegetal necesario para mejorar los índices de existencias actuales.

Según Margaret (1991), la competencia existente entre individuos de una misma especie, Competencia Intraespecifica, es un factor im- portante en el mantenimiento de la diversidad del bosque; en el bosque tropical lluvioso se encuentra que los árboles maduros deprimen el desarrollo de plántulas de su propia especie, pero a su vez, son capaces de crecer en proximidad estrecha con plántulas de otras especies, siempre que otros factores no interfieran.

Gracias a los estudios de poblaciones, es posible estudiar el comportamiento del número de individuos existente en cada clase diamétrica para una determinada especie. La distribución diamétrica de varias especies forestales tropicales, en bosques naturales, es conocida como 
"Distribución Diamétrica en J invertida" (Hutchings, 1997; Palacios, 2002). Presenta un alto número de individuos en la primera clase, pero una drástica disminución de ellos al pasar a la categoría siguiente.

La iniciativa de estudiar la regeneración de las especies en estudio, fue escuchada, respaldada y mejorada por nuestros compañeros de investigación. Las observaciones y conversaciones tenidas durante los recorridos, enriquecieron y permitieron dar forma a esta fase de la investigación. Ellos señalaban basados en su conocimiento tradicional y su constante observación, que generalmente, cerca de grandes árboles de determinada especie, se encontraban por épocas un alto número de plántulas, pero que poco a poco iban muriendo, hasta que finalmente muy pocos lograban sobrevivir.

- Buscando buenas madres... Selección de fuentes semilleras

Para comenzar fue necesario realizar una serie de recorridos por diferentes lugares buscando individuos de las especies seleccionadas. Se inició con los árboles madres utilizados para el ensayo de reproducción por estacas, pero si queríamos tener una idea más acertada de cómo era la regeneración natural en esas especies, debíamos aumentar el número de árboles observados. Se visitaron un mínimo de 7 árboles maduros por especie en los cuales realizamos una breve caracterización del hábitat en que se desarrollan y un diagnóstico de su regeneración.

- Conociendo la casa donde crecen...Caracterización ecológica de los hábitats

Cuando se identificaba un árbol madre, lo que inicialmente realizábamos era una caracteriza- ción básica del hábitat en donde crecen las plántulas. Determinábamos qué tipo de bosque era, qué otras especies crecían cerca de esos árboles madres, la altura promedio del dosel, los animales que lo visitaban y hasta donde fuera posible conocer la historia de ese bosque, si habían sacado madera, o hecho chagra, o si alguna familia había vivido cerca de ese lugar. La caracterización general del hábitat donde se encuentran las especies en estudio se presenta en la Tabla 9.

\section{- Contando sus crías de ellos Diagnósticos de regeneración}

Sostenidos en los aspectos de competencia intraespecífica, distribución diamétrica y las observaciones de los investigadores locales, nuestra idea era evaluar el número de plántulas que se desarrollan alrededor del árbol madre y determinar si es factible obtener una cantidad considerable de ellas para luego en un momento determinado, antes que se inicie la mortalidad, bloquearlas, y llevarlas al vivero para repoblar otras partes del bosque con éstas.

Con el conocimiento que tenían sobre las especies los investigadores locales y los externos, diseñamos un tipo de diagnóstico de regeneración natural basándonos en las características de los frutos, semillas y sus fenómenos de frugivoría y dispersión. Fue un modelo sencillo y bastante práctico, en el cual se contaban todas las plántulas encontradas debajo y hasta cinco metros fuera de la copa del árbol. Por lo general, todas las plántulas que se encontraron bajo el árbol madre tenían un tamaño similar. Los inventarios de regeneración realizados a cada árbol madre de las especies, junto con algunos aspectos de sanidad, competencia y desarrollo de las plántulas a continuación se señalan (Tabla 10). 
Tabla 9. Caracterización básica de los hábitats de las especies en estudio.

\begin{tabular}{|c|c|}
\hline ESPECIE & TIPO DE BOSQUE \\
\hline 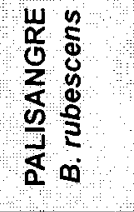 & $\begin{array}{l}\text { Selva de tierra firme, poco intervenida. Altura del dosel entre } 25-40 \mathrm{~m} \text { Los individuos adultos de esta espe- } \\
\text { cie son dominantes en el dosel. Se encontraron cerca Muena (Ocotea sp), Acapú (Minqartia guianensis), } \\
\text { Caimitilo (Sapotaceae), Cumala de la alta (Virola sp.) y palma carana. Varios individuos de esta especie se } \\
\text { caen debido a la acción de los fuertes vientos y a que no desarrolla grandes bambas que puedan ayudarle } \\
\text { a controlar su peso. }\end{array}$ \\
\hline 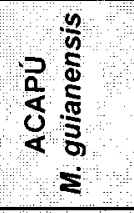 & $\begin{array}{l}\text { Selva de tierra firme, poco intervenida. Altura del dosel entre } 20-30 \text { m. Sus individuos son dominantes y } \\
\text { codominantes en el dosel. Generalmente se encontraron alrededor Castaño (Couroupita sp.), Pona lisa } \\
\text { (Socratea sp.) y Pona barrigona (Iriartella sp) y en el sotobosque algunas especies de melastomataceas y } \\
\text { rubiaceas. En algunas partes del bosque es posible encontrar manchales naturales de esta especie. }\end{array}$ \\
\hline 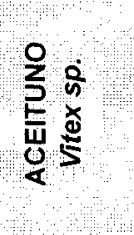 & $\begin{array}{l}\text { Se desarrolla en bosques inundables, por lo general cerca de un caño o quebrada, o en la cabecera de } \\
\text { alguna quebrada. Los investigadores locales señalan que es posible encontrar manchales de esta especie } \\
\text { y que a veces también en bosques secundarios o claros. Altura del dosel entre } 20-30 \text {. Sus individuos son } \\
\text { generalmente los más dominantes. Generalmente se encuentran Castaño (Couroupita sp.), Palma chapaja } \\
\text { (Scheeleasp.), huicungo (Astrocaryum sp), algunas apocináceas. Nunca se encontró regeneración natural } \\
\text { de esta especie, ni se observaron individuos en fructificación. }\end{array}$ \\
\hline$\frac{5}{2} \sum_{0}^{\frac{\pi}{\pi}}$ & $\begin{array}{l}\text { Selva de tierra firme y también existe un tipo de quinilla en la selva inundable Se diferencian en la cantidad } \\
\text { de duramen su madera, es mayor en la quinilla de tierra firme. Altura de dosel entre } 20-40 \mathrm{~m} \text {. Sus indivi- } \\
\text { duos están generalmente en el estrato dominante y codominante del dosel. Los micos churuco (Lagothryx } \\
\text { lagothicha), cotudo (Aluatta sp.) y el murciélago chimbilaco (Chiroptera: Phyllostomidae) son los encarga- } \\
\text { dos de la dispersión de sus semillas. Generalmente se encuentran cerca matamata (Eschweilera spp.), } \\
\text { Acapú (Minquartia guianensis). }\end{array}$ \\
\hline$\frac{\pi}{3} \frac{\pi}{\frac{2}{2}}$ & $\begin{array}{l}\text { Se encuentra generalmente en bosques con edades sucesionales tempranas, o en selvas poco interve- } \\
\text { nidas pero cerca de la cabecera de una quebrada, Sus individuos se encuentran en el estrato dominante } \\
\text { Altura del dosel entre } 15-25 \mathrm{~m} \text {. Durante los diagnósticos de regeneración no se observaron otras especies } \\
\text { asociadas; sin embargo, los investigadores locales recalcan la importancia de este árbol dentro de los } \\
\text { bosques, principalmente como fuente alimenticia para muchos animales y aves. }\end{array}$ \\
\hline
\end{tabular}

Tabla 10. Diagnósticos de Regeneración Natural de las especies en estudio.

\begin{tabular}{|c|c|c|c|c|c|}
\hline ESPECIE & $\begin{array}{l}\text { A. Madres } \\
\text { Total }\end{array}$ & $\begin{array}{l}\text { A. Madres } \\
\text { con Rgh }\end{array}$ & $\begin{array}{c}\text { DAP rango } \\
(\mathrm{cm})\end{array}$ & To Plant & OBSERVACIONES \\
\hline $\begin{array}{l}\text { ACAPU } \\
\text { M. guianensis }\end{array}$ & 10 & 6 & $25-70$ & 59 & $\begin{array}{l}\text { Algunos individuos presentaron gran } \\
\text { cantidad de semilla verde }\end{array}$ \\
\hline $\begin{array}{l}\text { ACEITUNO } \\
\text { Vitex sp. }\end{array}$ & 8 & 2 & $30-100$ & 13 & $\begin{array}{l}\text { Se observa por lo general rebrotes en } \\
\text { troncos caidos }\end{array}$ \\
\hline \begin{tabular}{|l|} 
QUINILLA \\
M. bidentata \\
\end{tabular} & 11 & 3 & $20-100$ & 4 & $\begin{array}{l}\text { Es comun observar entre diciembre y } \\
\text { enero una gran cantidad de RgN }\end{array}$ \\
\hline $\begin{array}{l}\text { PALISANGRE } \\
\text { B rubescens }\end{array}$ & 7 & 5 & $65-100$ & 68 & $\begin{array}{l}\text { Es comun observar varios troncos de } \\
\text { arboles volcados por accion de } \\
\text { vientos }\end{array}$ \\
\hline OJE & $9:$ & 0 & $20-50$ & 0 & $\begin{array}{l}\text { Sus frutos son dispersados por aves y } \\
\text { murcielagos por tal razon no se } \\
\text { encontro rgn con el metodo utilizado }\end{array}$ \\
\hline $\begin{array}{l}\text { CEDRO } \\
\text { Cedrela sp. }\end{array}$ & 4 & 2 & $20-25$ & 18 & $\begin{array}{l}\text { Muy pocos individuos con diametros } \\
\text { mayores a } 40 \mathrm{~cm}\end{array}$ \\
\hline $\begin{array}{l}\text { CASTAÑO } \\
\text { Lecythis sp. }\end{array}$ & 3 & 3 & $70-50$ & 87 & $\begin{array}{l}\text { La madera de esta especie se utiliza } \\
\text { en la construcción de casas }\end{array}$ \\
\hline
\end{tabular}

A. Madres Total: Número total de árboles madres inventariados. A. Madres con Rgn: Número total de árboles madres en las que se encontró regeneración natural. No. Plantas: Número promedio de plántulas en un árbol con regeneración. 
- Llevándolas al vivero Ensayos de trasplante

Con el objetivo de observar la capacidad de resistencia al trasplante y al cambio de condiciones ambientales de las especies en estudio, al realizar los inventarios de regeneración natural, trasplantamos algunas plántulas del bosque de las especies que presentaron un número mayor a 100 plántulas.

Trasplantamos 200 plántulas de dos especies: Acapú ( $M$. guianensis), y Palisangre (B. rubescens), 100 de cada una. En el bloqueo se llevaron las plántulas con un pan de tierra $(1 / 201 / 4 \mathrm{de}$ una bolsa para vivero), para cuidar sus pequeñas raíces y tener algunos posibles microorganismos benéficos para el desarrollo de éstas.

La manera de transportar las plántulas erạ un paso crítico en la investigación, ya que dentro del monte el único medio es el hombre; se debía hacer de tal manera que se lastimaran lo menos posible. Se empacaron las plántulas en costales de fique, cada uno contenía 50 de ellas, luego se amarraban con soga a la cabeza y se recostaban en la espalda. Así se transportaban dentro del monte hasta el lugar donde se encontraba la canoa, luego se llevaban en ella hasta un puerto de la comunidad y luego se llevaban de nuevo amarrados a la cabeza hasta el vivero.

Al día siguiente se llenaban completamente las bolsas de las plántulas, con un sustrato de $a b o-$ no de palo muerto y aserrín, y se dejaron en una era del vivero (Figura 4). Luego nuestras crías entraron en "cuidados intensivos" durante 15 días donde se definiría la tasa de mortalidad de cada especie. Los resultados obtenidos luego de 15 días de trasplantadas al igual que los obtenidos luego de 35 días se presentan en la Tabla 11.

Tabla 11. Tasas de sobrevivencia y mortalidad en los ensayos de trasplante.

\begin{tabular}{|c|c|c|c|c|c|}
\hline \multirow{2}{*}{ ESPECIE } & \multicolumn{2}{|c|}{ Sobrevivencia (\%) } & \multicolumn{2}{c|}{ Mortalidad $(\%)$} & \multirow{2}{*}{ Observaciones } \\
\cline { 2 - 6 } & 15 días & 35 días & 15 días & 35 días & Hojas nuevas, en la se- \\
Palisangre & 75 & 75 & 25 & 25 & gunda evaluación. \\
\hline Acapú & 97 & 97 & 3 & 3 & \\
\hline
\end{tabular}
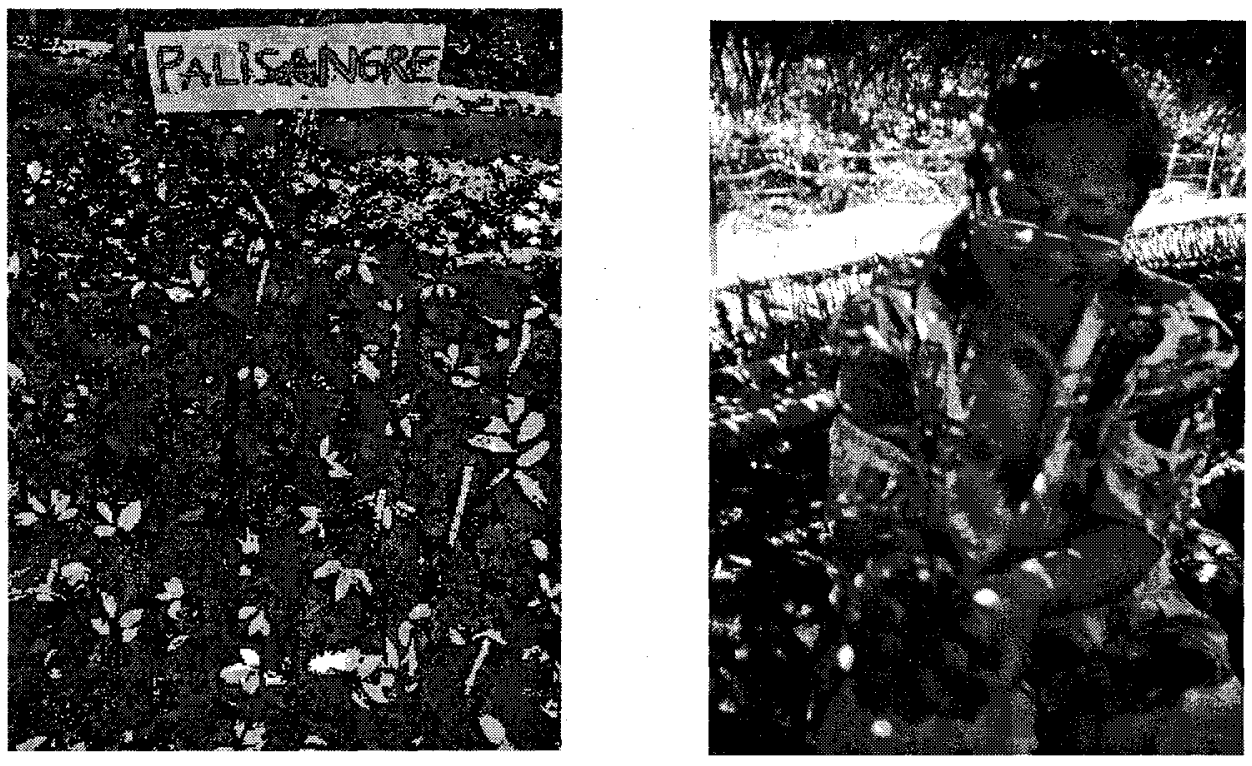

Figuras 4. Ensayos de trasplante. Foto N. Lozano. 2004 
Las tasas de mortalidad encontradas no son altas, especialmente en el acapú, tomando en cuenta que fue el primer ensayo de bloqueo de regeneración natural que se ha realizado para estas especies en la región, además de lo difícil que fue el transporte y el cambio ambiental al cual se sometieron las plántulas. Consideramos que la consecución de material vegetal de palisangre y acapú por el método silvicultural de regeneración natural ofrece grandes ventajas frente a la regeneración artificial de éstas, ya que se logra obtener plántulas totalmente adaptadas a las condiciones del sitio, al tiempo que se aprovechan de una mejor manera los recursos disponibles en el bosque natural como son: sustrato, microorganismos benéficos, control natural, agua, brillo solar, etc.

Realmente son pocas las investigaciones que se han realizado en regeneración natural de especies maderables tropicales. Sin embargo, es gratamente sorprendente conocer el impacto positivo que pueden tener las investigaciones silviculturales de regeneración natural, en el desarrollo de nuevas pautas y prácticas de manejo que mejoren la actual situación de los bosques tropicales; por ejemplo, en el proyecto Manglares de Colombia (Sánchez y Ulloa, 1999, Sánchez et al., 2004), luego de caracterizar este ecosistema se realizaron prácticas de restauración en áreas degradadas, utilizando plántulas provenientes tanto del bosque natural como de viveros comunitarios. También en Bolivia y Costa Rica se han generado metodologías para desarrollar técnicas de manejo del bosque basadas en la regeneración natural de algunas especies maderables (Vega, 1997; CATIE, 2001).

La actual investigación, a pesar del corto tiempo de duración, permite orientar y motivar futuros proyectos en regeneración natural de especies maderables de la Amazonia colombiana que contribuyan a un mejor conocimiento $\mathrm{y}$ manejo de nuestros bosques naturales.
EVALUACIÓN FINAL DEL PROYECTO

Después de cinco meses de trabajo, el grupo de investigación realizó una reunión final para hacer un recuento y análisis del camino construido. Los actores locales enunciaron dos razones principales que demuestran la importancia de este trabajo para la comunidad; éstas se presentan en las conclusiones finales del artículo y principalmente tienen que ver con lo que Pinkerton (2002) llama desarrollo de capacidades y habilidades locales, que es una de las características de la silvicultura holística.

Los pequeños callos, raíces y rebrotes foliares de las diferentes especies, las plántulas trasplantadas del bosque, la construcción de un vivero comunitario, entre otros, son elementos que permiten al grupo de investigación tener una mayor confianza en el trabajo realizado por el mismo. Además, este trabajo es un avance en la construcción del conocimiento de manera conjunta, se constituye en una propuesta integradora de conocimientos y prácticas de manejo, tradicionales y occidentales que, como lo indica Folke et al. (2002), ofrecen una ayuda para contrarrestar algunas de las actuales crisis relacionadas con el manejo convencional de los recursos naturales.

La realización del informe final se llevó a cabo en Bogotá; luego éste fue llevado de nuevo a la comunidad y allá, en conjunto con los investigadores locales, se discutió su pertinencia, sus alcances y principalmente la necesidad de continuar con el proceso de investigación. Esta es una característica típica de los proyectos realizados bajo la Investigación Participativa, donde la etapa final de los proyectos es al mismo tiempo el inicio de uno nuevo, es decir, el proceso de investigación sigue el ritmo de una espiral en ascenso que busca finalmente el empoderamiento y la autogestión de las comunidades locales.

Los resultados finales del proyecto fueron socializados por los mismos investigadores loca- 
les a toda la comunidad y a algunos representantes de las comunidades vecinas, Vergel, Zaragoza, Palmeras, Macedonia y San Martín. De esta manera se demostró aún más la apropiación del proyecto por parte de los habitàntes locales.

Finalmente, los resultados se presentaron al Grupo de Trabajo en Investigación (GTI) conformado por funcionarios del PNNA y representantes de las seis comunidades indígenas asentadas dentro de este (PNN Amacayacu, 2003) y allí, con el ánimo de recoger las iniciativas locales surgidas, continuar con el proceso de investigación participativa e involucrar a las otras comunidades que se encuentran dentro del PNNA. El GTI adoptó la idea de seguir con este proyecto para producir y sembrar plántulas de especies maderables usadas por las comunidades locales, buscando recuperar zonas degradadas, aumentar las existencias actuales de árboles con importancia local y aportar al mejoramiento de la calidad de vida de estas comunidades indígenas.

\section{CONCLUSIONES Y RECOMENDACIONES}

- Los avances y resultados que se obtuvieron en esta investigación se deben principalmente al tipo de integrantes del grupo de trabajo. El "aprender-haciendo" y el poder combinar los conocimientos técnicos con ese enorme conocimiento indígena, han permitido crear nuevas propuestas en el manejo y propagación de especies forestales con importancia en las comunidades asentadas dentro del PNN Amacayacu y, así mismo, se avanzó en el objetivo de utilizar sosteniblemente los recursos naturales existentes en el Parque y se inició un proceso de investigación participativa relacionado con el uso y manejo sostenible de los recursos forestales de la región.

- Es importante realizar investigaciones participativas que den verdaderamente un papel activo a los actores locales, buscando su apropiación del proyecto, pues de esta manera, además de enriquecer la investigación, se realiza un diálogo de saberes donde es posible avanzar en la construcción de nuevos conocimientos como lo sugieren Folke et al. (2002), pueden contrarrestar algunas de las actuales crisis relacionadas con el manejo convencional de los recursos naturales.

- El mejor tratamiento y medio para el enraizamiento de estacas de aceituno (Vitex $s p$ ) es inmersión de las estacas en hormonagro (5000 ppm) durante 15 minutos, sembrando en el sustrato 1 suelo + 1arena + 1aserrín, obteniendo de esta manera resultados del $97-100 \%$.

- Para obtener en palisangre (Brosimum rubescens) un desarrollo del sistema radicular alrededor del $30 \%$, se recomienda trabajar con estacas de diámetro entre 2$4 \mathrm{~cm}$, con inmersión en hormonas (5000 ppm, durante 15 minutos), realizando la siembra en cualquiera de los dos sustratos; sin embargo, para acelerar la formación de raíces se recomienda el sustrato dos, que presenta unas características similares al suelo de la región.

- El tratamiento que presentó mejores resultados $(40 \%)$ en el desarrollo radicular de la quinilla (Manilkara bidentata) consiste en emplear estacas de diámetro entre $2-4 \mathrm{~cm}$, sin inmersión en auxinas, sembrando en el sustrato 1suelo + 1arena + 1aserrín.

- El ojé (Ficus insipida) a pesar de haber sido la primera especie en mostrar resultados, debido a las condiciones de la era de propagación y a la suculencia de las estacas, sufrió un ataque de hongos, lo que hizo necesario desmontar el ensayo previniendo así la presencia de la enfermedad en los otros ensayos. Por otra parte, el acapú ( $M$. guianensis) fue la única especie que no arrojó resultado alguno; sin embargo, el 
estado de las estacas al mes de establecido el ensayo era bueno, puesto que se encontraban carnosas y con abundante exudado al realizar pruebas de corte; indicando esto la posibilidad de la especie de propagarse asexualmente, pero en un lapso de tiempo mayor.

- Con los resultados obtenidos durante este proyecto, es posible sugerir que el manejo de regeneración natural es el mejor método para obtener individuos de palisangre y acapú; mientras que para el aceituno, el mejor método de producción es la propagación vegetativa por estacas, y para la quinilla, el método más adecuado según el conocimiento de los habitantes locales es la propagación sexual. No obstante, el grupo de investigación considera necesario realizar los ensayos de reproducción sexual y continuar con el trasplante de plántulas y los diagnósticos de regeneración natural, con el fin de evaluar la oferta de frutos, semillas y regeneración natural de las especies en estudio en otra época del año.

- Los actores locales enunciaron dos razones principales que demuestran la importancia de este trabajo para la comunidad, especialmente como lo señala Pinkerton (2002), en lo que tiene que ver con el desarrollo de capacidades y habilidades locales:

"Este proyecto es un avance en la recuperación de nuestras áreas que han perdido sus especies maderables..."

"Es importante la investigación porque por medio de nosotros la comunidad va a saber cómo se hace un vivero con todas las técnicas..."

- Este proyecto generó en la comunidad, especialmente en los investigadores locales, iniciativas e ideas en torno a nuevas investigaciones que continúen y mejoren los resultados obtenidos. En este orden de ideas y luego de la presentación de los resultados a diferentes actores de la región, las demás comunidades indígenas asentadas dentro del PNN Amacayacu y el GTI, adoptaron la iniciativa de producir plántulas de especies maderables con importancia local y realizar siembras comunitarias con el ánimo de aumentar las actuales existencias de dichas especies, recuperar zonas degradadas $y$, por ende, mejorar su calidad de vida. Esta es una característica típica de los proyectos realizados bajo la Investigación Participativa, donde la etapa final de los proyectos es al mismo tiempo el inicio de uno nuevo, es decir, el proceso de investigación sigue el ritmo de una espiral en ascenso que busca finalmente el empoderamiento y la autogestión de las comunidades locales.

\section{AGRADECIMIENTOS}

Queremos expresar nuestros más sinceros agradecimientos a la Fundación TropenbosColombia por el apoyo logístico y financiero; pero especialmente a Carlos Rodríguez, Rosa M., Amanda, Paula y Christine por la confianza y apoyo dados en todo momento. A la UAESPNN por permitirnos desarrollar el proyecto; especialmente a José Sinisterra, por su apoyo y orientación. A todos los funcionarios del PNNA por estar siempre dispuestos a colaborarnos. A los profesores de la Universidad Distrital Max Triana y Heliodoro Sánchez por su apoyo y amistad.

Al GTI, y especialmente a Sara Bennett por sus sabios consejos. A la comunidad indígena de Mocagua, por recibirnos tan bien y'considerarnos como sus hijos. A todos los integrantes del grupo dé investigación, pues gracias a ellos el proyecto se llevó a cabo. A la familia Vásquez por abrir las puertas de su hogar para nosotros. A Alberto Parente y su familia por apoyarnos y brindarnos su amistad. $\mathrm{Y}$ a todas aquellas personas que con sus consejos y orientaciones hicieron parte de este proyecto. 


\section{REFERENCIAS BIBLIOGRÁFICAS}

ARIAS J. 1997. Propuestas para un desarrollo sostenible en el Parque Nacional Amacàyacu. En Seminario sobre alternativas de desarrollo económico para la producción local de espacios naturales protegidos. FPNNE. Madrid, España.

CATIE. 2001. Silvicultura de bosques húmedos con énfasis en América Central. Manual Técnico del CATIE No 46. Turrialba, Costa Rica.

CORTÉS, E. 1983. Ensayo de propagación por estacas de Tabebuia rosea (Guayacán), Brosimum utile (Sande) y Virola sebifera (Sangre toro). INDERENA. Ministerio de Agricultura. Bogotá.

DÍAZ, L. 1986. Estudio de propagación vegetativa por estacas de cinco especies forestales de bosque muy húmedo tropical, vivero de San Isidro (Quibdó, Chocó). Bogotá. Trabajo de grado (Ingeniero Forestal). Universidad Distrital Francisco José de Caldas. Facultad del Medio Ambiente y Recursos Naturales.

FOLKE, CARL., FIKRET B., COLDING, J. 2002. Ecological practices and social mechanisms for building resilience and sustainability. En: Berkes, F., Folke C., 2002. Linking Social and Ecological Systems. Management practices and social mechanisms for building resilience. Cambridge University Press.

FRANCO, J. 2002. Etnobotánica de la yanchama (Ficus spp. Moraceae) Amazonas, Colombia. Bogotá. Trabajo de grado (Biólogo). Universidad Javeriana.

GARZÓN, C. \& MACURITOFE, V. 1992. La Noche, las Plantas y sus Dueños: aproximación al conocimiento botánico en una cultura amazónica. Corporación Colombiana para la Amazonia Araracuara. Editorial Gente Nueva.

HARTMAN, H \& KESTER, D. 1987. Propagación de plantas, principios y prácticas. Compañía editorial Continental. México.
HUTCHINGS, M. 1997. The Structure of Plant Populations. En: Crawley, M. (ed.). Plant Ecology. Blackwell Science Ltd. New York.

IGAC. 1995. Suelos de Colombia; origen, evolución, clasificación, distribución y uso. IGAC. Bogotá.

LA ROTTA C. 1987. La etnobotánica: una aproximación metodológica desarrollada con la comunidad indígena Miraña (AmazonasColombia). En: Memorias del primer simposio colombiano de etnobotánica. Corporación de Araracuara. Editorial Gente Nueva. Bogotá.

MARGARET, L. 1991. Ecología de Plantas Tropicales. Editorial Limusa. Mexico.

NAVARRO, R \& PEMÁN, J. 1997. Apuntes de producción de planta forestal. Universidad de Córdoba. España.

PALACIOS, P. et al. 2002. Estructura, densidad y patrones de distribución espacial, de las poblaciones naturales de $B$. rubescens. En: $\mathrm{Pa}-$ lacios et al., 2003. Valoración de la oferta ambiental de Palosangre (Brosimum rubescens Taubert), como especie promisoria en la elaboración de artesanías, en el sur del Trapecio Amazónico. Universidad Nacional de Colombia - COLCIENCIAS. Bogotá.

PARQUE NACIONAL NATURAL AMACAYACU. Equipo Humano. 2002. El manejo concertado de los bienes comunes: un ejercicio de soberanía en la Amazonia Colombiana. En: Ochoa, D. \& Guio, C. 2002. Control social y coordinación: un camino hacia la sostenibilidad amazónica. Caso de maderas del trópico amazónico. Defensoría del Pueblo. U. Nacional. UEASSPNN. Colombia.

PINKERTON E. 2002. Integrated management of a temperate montane forest ecosystem through wholistic forestry: a British Columbia example. En: Berkes, F., Folke C., 2002. Linking Social and Ecological Systems. Management practices and social mechanisms for building resilience. Cambridge University Press. 
SÁNCHEZ, H., ULLOA, G. 1999. Experiencias de restauración en el proyecto Manglares de Colombia. En: GTZ, Fundación Alejandro Ángel Escobar, FESCOL. Memoriàs del seminario de restauración ecológica y reforestación. Bogotá.

SÁNCHEZ, H., ULLOA, G., TAVERA, H. 2004. Manejo Integral de los manglares por comunidades locales. Caribe de Colombia. Ministerio de Ambiente, Vivienda y Desarrollo Territorial., CONIF., OIMT. Bogotá.

SUÁREZ M, et al., 1998. Estrategias para el desarrollo de las comunidades indígenas Tikuna en el Parque Nacional Natural Amacayacu,
Amazonas-Colombia. Ponencia presentada en el Primer Congreso Latinoamericano de Parques Nacionales y otras Áreas Protegidas. Santa Marta, Colombia.

TRUJILLO, E. 1999. Manejo de semillas, viveros y plantación inicial. Editado por CEDETRABAJO. Bogotá.

VEGA, L. 1997. Pautas sobre una metodología para desarrollar técnicas de manejo mediante regeneración natural del bosque tropical mixto en Bolivia. En: Simposio Internacional "Posibilidades de manejo forestal sostenible en América tropical". Santacruz de la Sierra, Bolivia. 